\title{
BMJ Open How do patients and the public understand overtesting and overdiagnosis? A protocol for a thematic meta-synthesis of qualitative research
}

\author{
Tomas Rozbroj (D) , ,2 Romi Haas, ${ }^{1,2}$ Denise A O'Connor, ${ }^{1,2}$ Rae Thomas (D) , \\ Kirsten McCaffery, ${ }^{4}$ Stacy Carter (D) , ${ }^{5}$ Rachelle Buchbinder ${ }^{1,2}$
}

To cite: Rozbroj T, Haas $R$, O'Connor DA, et al. How do patients and the public understand overtesting and overdiagnosis? A protocol for a thematic meta-synthesis of qualitative research. BMJ Open 2020;10:e037283. doi:10.1136/ bmjopen-2020-037283

- Prepublication history and additional material for this paper are available online. To view these files, please visit the journal online (http://dx.doi org/10.1136/bmjopen-2020037283).

Received 27 January 2020 Revised 13 May 2020 Accepted 20 May 2020
Check for updates

(C) Author(s) (or their employer(s)) 2020. Re-use permitted under CC BY-NC. No commercial re-use. See rights and permissions. Published by BMJ.

For numbered affiliations see end of article.

Correspondence to

Dr Tomas Rozbroj;

tomas.rozbroj@monash.edu

\section{ABSTRACT}

Introduction Examining patient and public understanding of overtesting and overdiagnosis (OverTD) is vital for reducing the burden of OverTD. Studies from disparate contexts, disciplines and focusing on disparate healthcare issues have examined patient and public understanding of OverTD. A synthesis is needed to bring this literature together, examine common themes, strengthen conclusions and identify gaps. This will help steer further research, policy and practice to improve patient and public understanding of OverTD. The objective of this study is to synthesise qualitative research data about patient and public understanding of OverTD.

Methods and analysis A thematic meta-synthesis will be used to synthesise primary qualitative research and qualitative components of primary mixed-methods research about patient and public understanding of OverTD. Studies published in English will be included. These will be identified using systematic searches from inception to March 2020 in the Scopus, CINAHL, PsycINF0 and MEDLINE databases. Studies that satisfy eligibility criteria will be assessed for methodological quality using the Critical Appraisal Skills Programme (CASP) checklist. Thematic meta-synthesis will comprise three stages: (1) line-by-line coding; (2) generation of descriptive themes and (3) generation of analytic themes. Confidence in the synthesis findings will be assessed using the Grading of Recommendations Assessment, Development and Evaluation Confidence in Evidence (GRADE CERQual) approach. A summary of GRADE CERQual results will be presented alongside the key themes. Study eligibility screening, data extraction, analysis and the CASP and GRADE CERQual assessments will be undertaken independently by two review authors.

Ethics and dissemination Ethics approval is not required for this secondary analysis of published data. The results will be disseminated in peer-reviewed journals and may be presented in conference papers and elsewhere. PROSPERO registration number CRD42020156838

\section{INTRODUCTION}

The high prevalence of overtesting, overdiagnosis and overtreatment across a range of health conditions is a global challenge. ${ }^{1}$ Overtesting is when diagnostic tests that are
Strengths and limitations of this study

- The first meta-synthesis of qualitative research about patient and public understanding of overtesting and overdiagnosis.

- Systematic search strategy informed by up-to-date evidence about database and keyword optimisation.

- Confidence in the qualitative meta-synthesis findings strengthened by use of the Grading of Recommendations Assessment, Development and Evaluation Confidence in Evidence approach.

- Scope of the research limited by the exclusion of studies not written in the English language and of grey literature.

not indicated are used. ${ }^{2}$ It can lead to overdiagnosis, ${ }^{3}$ which is when a diagnosis is made according to professional standards, but when it is unlikely to benefit the patient. ${ }^{4}$ Overtesting and overdiagnosis (OverTD) can lead to overtreatment, ${ }^{25}$ which is treatment that does more harm than good. ${ }^{6}$

It is important to reduce overtesting, overdiagnosis and overtreatment. ${ }^{78}$ Overtesting can lead to harms including unnecessary invasive procedures, false positives and misdiagnoses. ${ }^{2}$ Overdiagnosis can lead to unwanted behavioural and psychological responses in patients, such as reduced participation in usual activities, ${ }^{9}$ stress and anxiety. ${ }^{10} \mathrm{~A}$ diagnosis primes patients and physicians to commence treatment, even for benign conditions. ${ }^{6}$ Overtreatment can lead to patient suffering, treatment-related complications, loss of quality of life, lost productivity and other burdens ${ }^{612}$ Medical overuse is massively costly to healthcare systems and to patients and their families, ${ }^{21314}$ and must be reduced to maintain healthcare system sustainability. ${ }^{15}$

Improving patient and public understanding of OverTD is key to reducing their incidence as well as the incidence of 
overtreatment. ${ }^{1516}$ Both patients and the public need to be aware of OverTD, as people regularly transition between being in and out of medical care, ${ }^{17}$ and their medical decision making is informed by beliefs that are formed and reformed across contexts. ${ }^{18}$ Presently, patients and the public often drive medical overuse. Some patients and members of the public tend to over-rely on tests and diagnoses, ${ }^{19}$ overestimating their benefits, ${ }^{20}{ }^{21}$ underestimating their risks ${ }^{22}$ as they cope with uncertainty poorly. ${ }^{323}$ Few are aware that overtesting or overdiagnosis occurs, ${ }^{24} 25$ and those who are often find the phenomena difficult to understand. ${ }^{25}{ }^{26}$ Research suggests that patient outcomes would be improved if they understood OverTD better. ${ }^{27}{ }^{28}$ Patients with better knowledge about OverTD make more appropriate screening and treatment decisions. ${ }^{25}$ Patient knowledge also influences the tests and treatments prescribed by the medical practitioners, ${ }^{29}$ who in some cases overuse medical interventions. ${ }^{30} 31$ Patients and the public want to be informed about OverTD, ${ }^{21}$ and need to understand both risks and benefits of medical interventions in order to participate in shared decision making. ${ }^{32}$

Research is increasingly examining patient and public understanding of OverTD. ${ }^{33}$ Patient and public understandings of OverTD have been surveyed, ${ }^{24} 34$ and qualitatively examined, in relation to a range of conditions and in multiple contexts. ${ }^{21}{ }^{26-28} 35$ Researchers have studied the challenges of communicating about OverTD to the general public ${ }^{27} 3637$ as well as to particular patient groups, such as patients with low health literacy. ${ }^{38}$ Strategies are being developed to overcome these communication challenges. They include the development of decision aids, which inform patients about the risks as well as benefits of particular medical interventions, ${ }^{25} \mathrm{such}$ as breast cancer screening, ${ }^{39}$ and assist them in making evidence-backed healthcare decisions. ${ }^{40}$ Other research has focused on refining patient educational tools. This includes studying how different concepts of OverTD resonate with patients and the public, ${ }^{41}$ the effects of information about overdiagnosis on patient screening decisions, ${ }^{21}$ and studying patients' understandings of their own diagnoses. ${ }^{10}$ The use of mass media to reduce OverTD has also been studied, such as how media narratives can influence cancer screening decisions ${ }^{42}{ }^{43}$ or promote better management of back pain. ${ }^{44}$

Despite progress in research, important gaps in knowledge remain. ${ }^{1645} 46$ First, existing studies are scattered across disciplines, contexts and focus on disparate medical conditions. ${ }^{47}$ It is difficult to appraise the overall state of research or glean its collective insights. Second, while it is known that patients and the public find OverTD unintuitive, little is known about why. ${ }^{1548}$ A meta-synthesis of qualitative data from research examining patient and public understanding of OverTD will help address these gaps. It will systematise insights from disparate disciplines, contexts and topic areas by identifying descriptive themes in the body of literature. The synthesis will also identify analytic themes about the reasons for poor public and patient understanding of OverTD. These findings will inform future research by highlighting priority areas for further enquiry. An increased understanding about why patients and the public struggle to understand OverTD may inform the development of educational interventions and other practice to improve their understanding.

\section{OBJECTIVE}

The objective of this study is to synthesise data from qualitative research on patient and public understanding of OverTD.

\section{METHODS}

Thematic meta-synthesis will be used to examine primary qualitative research and qualitative components of mixed-methods research about patient and public understanding of OverTD.

The protocol is presented in accordance with the Preferred Reporting Items for Systematic review and Meta-Analysis Protocols (PRISMA-P) checklist ${ }^{49}$ (available in online supplementary appendix 1).

\section{Study selection criteria}

Study selection criteria and their rationale are described in table 1.

\section{Search methods}

The search process will comprise first an informal scoping stage to develop search strategies, and then a formal main stage to identify and collate eligible studies. The main stage will identify English language studies indexed in four databases from inception until March 2020.

The scoping stage will be exploratory. Its aims are to become familiar with the literature, refine search parameters, identify MeSH terms and keywords and test the preliminary search strategy.

The main stage will comprise the formal literature search. It will be informed by the scoping stage, by search strategy guidelines from the Cochrane Collaboration, ${ }^{50} 51$ guidelines for optimising database searches for medical qualitative research ${ }^{52}$ and guidelines for searching the individual databases used, such as for MEDLINE ${ }^{53}$ and PsycINFO. ${ }^{54}$ Search filters will be identified for each of the inclusion criteria. A subject librarian will contribute to the development of the search strategy.

The following databases will be used: Scopus, CINAHL, MEDLINE and PsycINFO. These were chosen because they are most likely to index studies about patient and public understanding of OverTD: social research (Scopus); medicine/public health/health communication research (MEDLINE, CINAHL); psychological research (PsycINFO) and generalist fields (Scopus). Database selection was also informed by research showing that Scopus, MEDLINE and CINAHL searches retrieve some of the largest numbers of qualitative health studies, and the largest number of qualitative health studies not listed 
Table 1 Study selection criteria

\begin{tabular}{l} 
Inclusion criterion \\
\hline Primary, published, peer-reviewed \\
studies
\end{tabular}

Studies examining understanding

\section{Rationale}

Restricting the synthesis to primary, published, peer-reviewed studies matches the aims of examining primary evidence.

Understanding is defined as objectual understanding: understanding of something, such as collection of ideas or a subject matter ${ }^{71}$. Studies examining participants' knowledge, perceptions, sentiments, values or experiential understanding will be included. This reflects that understanding can be developed through experiential learning ${ }^{72}$, emotional learning ${ }^{73}$ as well as abstract learning.

Among patients and/or the public

The synthesis will examine understanding among both patients and the public. People regularly transition between being one or the other ${ }^{17}$, and make diagnostic and screening decisions drawing on understanding they developed overtime and in either role. So, it is appropriate to examine understanding of OverTD among both groups.

It will be distinguished whether studies are about patients, the public or both. People have a differing engagement with health decision making when they are patients or the public ${ }^{74}$. To account for this, synthesis results for each group will be compared, and important intergroup differences will be considered in study outcomes.

Of overtesting and/or overdiagnosis (OverTD)

Studies about both overtesting (OT) and overdiagnosis (OD) will be included, as both are deeply interlinked and underpinned by common broader patient ideas about healthcare. However, understandings of OT and OD may differ. To account for this, studies will be classified based on whether they examine OT, OD or both. The synthesis results will be compared by these classifications, and important differences will be accounted for.

Synonymous concepts to overtesting and overdiagnosis will be included, such as 'over-detection' and 'overuse of diagnostic testing'. The term 'overdiagnosis' was popularised relatively recently ${ }^{75}$, but it was predated by earlier terms ${ }^{76}$, and it is important to capture these earlier studies.

Studies which did not explicitly aim to understand how to inform patients or the public about OverTD are outside the scope of this synthesis and will be excluded. Studies that only address overtreatment and not overtesting or overdiagnosis will also be excluded.

Either qualitative or mixed-methods study design

Mixed-methods studies will be included where their qualitative components can still be examined in the thematic meta-synthesis.

Quantitative components of mixed-methods studies will be excluded, as will studies where it is not possible to differentiate between quantitative and qualitative components of analysis.

Published in the English language

Only English language studies will be included, as the authors are English speakers, and relying on translations of non-English studies could introduce inaccuracies into the analysis.

$\begin{array}{ll}\text { Published in any year } & \text { There will be no date restrictions: older insights may still be relevant. } \\ \text { Conducted in any setting } & \begin{array}{l}\text { There will be no setting restrictions: studies from all settings may potentially contain transferable } \\ \text { insights about patient and public understandings of OverTD. }\end{array} \\ \begin{array}{ll}\text { Focusing on the general concepts } \\ \text { of OverTD and/or in relation to any }\end{array} & \begin{array}{l}\text { While patient and public understanding of OverTD may differ depending on medical conditions, } \\ \text { there may be underlying themes across conditions, so it is relevant to include studies relating to any } \\ \text { condition/s or interventions }\end{array} \\ \begin{array}{l}\text { conditions. The condition/s which a study focuses on will be noted. Study themes will be compared } \\ \text { by conditions in analysis if the sample characteristics make this viable. }\end{array}\end{array}$

by other databases. ${ }^{52}$ Additionally, PsycINFO was included despite indexing relatively few unique studies, ${ }^{52}$ because it may index studies about psychosocial factors related to understanding OverTD. Examples of all search strategies, including filters for each criterion and Boolean operators, are included in online supplementary appendix 2.

Even where database selection is optimised, one study shows that $7 \%$ of qualitative health studies that fit the search parameters will not be retrieved, with the majority not indexed by major databases. ${ }^{52}$ To increase the chances of relevant studies being retrieved, the reference lists of all studies included in the final sample will be scanned for eligibility, and experts in the field will be contacted to identify studies that may have been missed. Potentially eligible studies will be added to the data screening process (described below).

The search may be rerun and results updated at a future date if required (ie, after 12 months if study is not yet published).

\section{Selection of studies}

Study selection will comprise the following steps:

1. All study records identified using the search strategy will be extracted with a PDF of the study manuscript into EndNote reference management software.

2. Duplicate studies will be removed from the data.

3. Study titles and abstracts will be screened for eligibility by two authors (TR and RH) working independently. Eligible studies and studies where eligibility cannot be clearly determined from the abstract and title will be included for full-text review.

4. Full texts will be independently read and examined for eligibility by TR and RH using a standardised form. Ineligible studies will be screened out, and the reason for exclusion recorded. Eligible studies will be included in the analysis. Where the two authors cannot agree on eligibility after discussion, a third author (DAO) will judge whether the study should be included. 
5. The final sample of full text studies will be extracted to NVivo research software.

The screening process will be reported in a PRISMA diagram. ${ }^{55}$

\section{Data extraction}

The complete study manuscript will be extracted into NVivo. Analysis will be undertaken on the Results sections of manuscripts, including themes, subthemes and primary data as reported in the manuscripts, such as participant quotes. Primary data included in tables and sections of manuscripts may also be analysed.

For each study, a standardised data collection form will be completed to capture:

- Study details: authors, year of publication, journal in which study was published.

- Research question/s.

- Participants: sample size, demographic characteristics, whether they are patients and/or the public, methods of participant recruitment and selection.

- Setting: type/s of healthcare and/or conditions the study focused on, whether the study examined overtesting and/or overdiagnosis, country where study was completed, whether study was in urban or rural settings.

- Method of data collection (such as interview or survey).

- Method of data analysis (such as narrative analysis or discourse analysis).

These details will be added as classifying information to the extracted full text studies in NVivo.

\section{Assessment of quality of included studies}

The Critical Appraisal Skills Programme (CASP) qualitative checklis $\mathrm{t}^{56}$ will be used to systematically examine the reliability, validity and usefulness of individual studies in the synthesis. The 10-item checklist comprises nine fixed-response questions that can be answered: yes/can't tell/no ('yes' indicates a positive score), and one textresponse question. Two authors (TR and $\mathrm{RH}$ ) will independently complete the CASP checklist for each study, and any disagreements in scoring will be resolved by a third author (DAO). A summary of CASP checklist results will be reported as a table and interpreted in text.

\section{Synthesis and analysis}

A thematic meta-synthesis of the Results sections of manuscripts will be undertaken. Analysis will comprise three main stages ${ }^{57}$ : first, line-by-line coding; next, descriptive thematic development, and finally; analytical theme development.

The thematic meta-synthesis method was chosen for several reasons. It fits the gaps this research responds to: the descriptive phase will address the need to systematise insights from disparate disciplines, contexts and topic areas, while the analytical phase is an interpretive tool with which synthesised studies can be re-examined to study why patients and the public find OverTD so difficult to understand. Furthermore, thematic analysis is suitable for handling data from disparate contexts, ${ }^{58}$ which fits this synthesis where included studies are likely to be heterogeneous. Finally, thematic meta-synthesis is particularly suited to informing policy and practice,${ }^{59}$ which is an important consideration for this research. The synthesis assumes an objective idealist epistemic position. The synthesised studies are considered to convey something about reality, but this reality is conveyed through a subjective lens. ${ }^{60}$ This is also assumed for the findings of this synthesis.

The first stage of analysis will be line-by-line coding. Authors will familiarise themselves with the data. TR will inductively generate initial codes for ideas in the data, coding over several iterations until no new codes are needed to capture ideas. Single data fragments can be assigned multiple codes. Once TR is satisfied with the code frame, he will code the whole dataset, checking coding for data coverage and refining it as necessary. A second author $(\mathrm{RH})$ will check a randomly selected sample of $10 \%$ of coded data for coding accuracy. A disagreement score will be calculated, and disagreements discussed and resolved, drawing on the wider team if required. An agreement score of $85 \%$ or higher will be targeted. ${ }^{61}$ If the agreement score is low, reasons for this will be investigated, and line-by-line coding may need to be repeated.

The second stage of analysis will be the development of descriptive themes to organise existing ideas in the data. TR and RH will independently organise individual codes into broader themes. The two authors will then cooperate to develop one set of common descriptive themes, discussing them with the wider author group. Themes will be checked for data coverage and internal homogeneity. ${ }^{62}$ External heterogeneity will not be assessed, as this is problematic where individual data can be multicoded. Themes will be revised until their fit with data is optimised.

The third stage of analysis will be the development of analytical themes capturing the barriers and enablers to patient and public understanding of OverTD. This stage will be interpretative and will seek to generate new ideas. ${ }^{57} 63$ TR and RH will independently re-examine the data organised into descriptive themes to infer what the barriers and enablers to understanding OverTD are. ${ }^{64}$ This phase will rely on the authors' subjectivities, and the authors will take a reflexive approach to minimise problems in interpretation and improve transparency in analysis. ${ }^{65} \mathrm{TR}$ and $\mathrm{RH}$ will meet to compare their analytical themes. As part of researcher reflexivity, they will discuss the factors that led to their interpretations, including their assumptions, logical inferences and how their interpretations may have been shaped by the predetermined research aims. Researcher reflexivity will also be addressed in peer-reviewed publications resulting from this research, including consideration about the ways in which the authors' own positions could have influenced the study design, analysis and the interpretation of findings. TR and RH will determine the analytical themes, 
which will be discussed and finalised with the wider author group.

Descriptive and analytical thematic results will be compared across a range of classifying variables, such as whether data are from studies about patients/the public/both, and whether data are from studies investigating understanding of overtesting/overdiagnosis/ both. Notable comparative differences will be reported in the Results. Descriptive and analytical themes will be tabulated and paired with exemplary data fragments. A separate table will display how the data from each study is represented in the coding.

\section{Assessment of confidence in findings}

The Grading of Recommendations Assessment, Development and Evaluation- Confidence in Evidence from Reviews of Qualitative research (GRADE-CERQual) approach $^{66}$ will be used to assess confidence in the analytic synthesis results. GRADE-CERQual is used to consider four factors about studies contributing to review findings: (1) methodological limitations; (2) relevance; (3) adequacy of supporting data and (4) coherence. The overall confidence in each review finding (ie, for each theme generated) will be graded as: high, moderate, low or very low. GRADE-CERQual assessments will be undertaken independently by two authors (TR and RH). Any disagreements will be discussed until consensus is achieved. Review findings, the confidence judgement for each finding and an explanation of the judgement will be presented in a Summary of Qualitative Findings table.

\section{Assessment of methodological limitations}

Methodological limitations in the synthesis will be judged based on the aggregated CASP checklist results for all included studies (described earlier).

\section{Assessment of relevance}

Relevance is 'the extent to which the body of data from the primary studies supporting a review finding is applicable to the context specified in the review question' (Noyes et al, p53). ${ }^{67}$ Across synthesised studies contributing to each review finding, we will consider the years of publication, settings in which studies were conducted, target audiences and specificity of the findings. These will determine how relevant the body of synthesised studies is for developing knowledge about contemporary patient and public understanding of OverTD in general.

\section{Assessment of adequacy}

Adequacy is the quantity and richness of data contributing to a review finding. ${ }^{68}$ Quantity is defined as the number of studies or data fragments supporting a theme. Richness is defined as the extent to which themes are supported by detailed, qualitative descriptions. Both parameters will be considered to judge the adequacy of data for supporting each theme in the synthesis results.

\section{Assessment of coherence}

Coherence is 'how clear and cogent the fit is between the data from the primary studies and a review finding that synthesises that data' (Colvin et al, p35). ${ }^{69}$ To examine coherence, the synthesis themes will be compared against the results of individual synthesised studies, examining the extent to which the synthesis findings align with individual study findings.

\section{Patient and public involvement}

A health consumer advocate from the Consumer's Health Forum of Australia was consulted in the development of this protocol. They will advise on the interpretation of the synthesis results.

\section{RESULTS}

The Results will comprise two subsections:

1. The sample profile, describing classifying information about the synthesised studies.

2. The thematic meta-synthesis results. Both descriptive and analytical themes will be reported. The descriptive themes will form a minor part of the Results, summarised in a table and briefly interpreted in text. The analytical themes will form a main part of the Results, with all major analytical themes tabulated, described in text and paired with exemplary data fragments and GRADE-CERQual assessment findings.

The meta-synthesis will be reported in accordance with the enhancing transparency in reporting the synthesis of qualitative research statement. ${ }^{70}$

\section{ETHICS AND DISSEMINATION}

Ethics approval is not required for this secondary analysis of published data. The findings may be disseminated in peer-reviewed publications, conference papers and elsewhere.

\section{Author affiliations}

${ }^{1}$ Monash Department of Clinical Epidemiology, Cabrini Institute, Monash University, Clayton, Victoria, Australia

${ }^{2}$ Cabrini Institute, Cabrini Health, Malvern, Victoria, Australia

${ }^{3}$ Faculty of Health Sciences and Medicine, Bond University, Gold Coast, Queensland, Australia

${ }^{4}$ Sydney School of Public Health, Sydney Medical School, The University of Sydney, Sydney, NSW, Australia

${ }^{5}$ Australian Centre for Health Engagement, Evidence and Values, University of Wollongong, Wollongong, New South Wales, Australia

Twitter Romi Haas @drromihaas, Rae Thomas @rthomasEBP, Kirsten McCaffery @ kirstenmccaffer?lang=en, Stacy Carter @stacymcarter and Rachelle Buchbinder @ rachellebuchbin?lang=en

Contributors TR, DAO and RB conceived the study and wrote the first draft of the protocol. RH, RT, KM and SC contributed to refining the protocol design and preparing subsequent protocol drafts. All authors approved the submitted protocol and are accountable for its content.

Funding This work is supported by an Australian National Health and Medical Research Council (NHMRC) Programme grant (Using Healthcare Wisely: reducing inappropriate use of tests and treatments, (APP1113532). DA0 is supported by an NHMRC Translating Research into Practice (TRIP) Fellowship (APP1168749). RT is supported by an NHMRC Programme grant (\#1106452). KM is supported by an 
NHMRC Principle Research Fellowship (1121110). SC is supported by an NHMRC Centres for Research Excellence grant (APP1104136). RB is supported by an NHMRC Senior Principal Research Fellowship (APP1082138).

\section{Competing interests None declared.}

Patient consent for publication Not required.

Provenance and peer review Not commissioned; externally peer reviewed.

Open access This is an open access article distributed in accordance with the Creative Commons Attribution Non Commercial (CC BY-NC 4.0) license, which permits others to distribute, remix, adapt, build upon this work non-commercially, and license their derivative works on different terms, provided the original work is properly cited, appropriate credit is given, any changes made indicated, and the use is non-commercial. See: http://creativecommons.org/licenses/by-nc/4.0/.

\section{ORCID iDs}

Tomas Rozbroj http://orcid.org/0000-0002-3084-746X

Rae Thomas http://orcid.org/0000-0002-2165-5917

Stacy Carter http://orcid.org/0000-0003-2617-8694

\section{REFERENCES}

1 Moynihan R, Doust J, Henry D. Preventing overdiagnosis: how to stop harming the healthy. BMJ 2012;344:e3502.

2 Zhi M, Ding EL, Theisen-Toupal J, et al. The landscape of inappropriate laboratory testing: a 15-year meta-analysis. PLoS One 2013;8:e78962.

3 Morgan S, Coleman J. We live in testing times - teaching rational test ordering in general practice. Aust Fam Physician 2014;43:273-6.

4 Carter SM, Rogers W, Heath I, et al. The challenge of overdiagnosis begins with its definition. BMJ 2015;350:h869.

5 Esserman LJ, Thompson IM, Reid B. Overdiagnosis and overtreatment in cancer: an opportunity for improvement. JAMA 2013;310:797-8.

6 Welch HG. Overdiagnosed: making people sick in the pursuit of health. Beacon Press, 2011.

7 Moynihan R, Heneghan C, Godlee F. Too much medicine: from evidence to action. BMJ 2013;347:f7141.

8 Wiener RS, Schwartz LM, Woloshin S. When a test is too good: how CT pulmonary angiograms find pulmonary emboli that do not need to be found. BMJ 2013;347:f3368.

9 O'Keeffe M, George SZ, O'Sullivan PB, et al. Psychosocial factors in low back pain: letting go of our misconceptions can help management. Br J Sports Med 2019;53:bjsports-2018-099816

10 Davies L, Hendrickson CD, Hanson GS. Experience of US patients who Self-identify as having an overdiagnosed thyroid cancer: a qualitative analysis. JAMA Otolaryngol Head Neck Surg 2017;143:663-9.

11 Heath I. Role of fear in overdiagnosis and overtreatment--an essay by lona Heath. BMJ 2014;349:96123.

12 Schlesinger M, Grob R. Treating, fast and slow: Americans' understanding of and responses to low-value care. Milbank $Q$ 2017;95:70-116.

13 Bulliard J-L, Chiolero A. Screening and overdiagnosis: public health implications. Public Health Rev 2015;36:8

14 Evans RG. Waste, economists and American healthcare. Healthc Policy 2013;9:12-20.

15 McCaffery KJ, Jansen J, Scherer LD, et al. Walking the tightrope: communicating overdiagnosis in modern healthcare. BMJ 2016;352:i348.

16 Morgan DJet al. Update on medical overuse: a review. JAMA Internal Medicine 2019;2019.

17 OECD. Consultations with doctors, in Health at a Glance 2017: OECD Indicators. Paris: OECD Publishing, 2017.

18 Abraham C, Sheeran P. Understanding and changing health behaviour: From health beliefs to self-regulation, in Understanding and changing health behaviour. Psychology Press, 2013: 19-40.

19 Barry CA, Bradley CP, Britten N, et al. Patients' unvoiced agendas in general practice consultations: qualitative study. BMJ 2000;320:1246-50

20 Main CJ, Buchbinder R, Porcheret M, et al. Addressing patient beliefs and expectations in the consultation. Best Pract Res Clin Rheumatol 2010;24:219-25.

21 Waller J, Douglas E, Whitaker KL, et al. Women's responses to information about overdiagnosis in the UK breast cancer screening programme: a qualitative study. BMJ Open 2013;3:e002703.

22 Hoffmann TC, Del Mar C. Patients' Expectations of the Benefits and Harms of Treatments, Screening, and Tests: A Systematic Review
Patient Expectations of Treatments and TestsPatient Expectations of Treatments and Tests. JAMA Internal Medicine 2015;175:274-86.

23 Schapira MM, Aggarwal C, Akers S, et al. How patients view lung cancer screening. The role of uncertainty in medical decision making Ann Am Thorac Soc 2016;13:1969-76.

24 Waller J, Whitaker KL, Winstanley K, et al. A survey study of women's responses to information about overdiagnosis in breast cancer screening in Britain. Br J Cancer 2014;111:1831-5.

25 Hersch J, Barratt A, Jansen J, et al. Use of a decision aid including information on overdetection to support informed choice about breast cancer screening: a randomised controlled trial. Lancet 2015:385:1642-52.

26 Moynihan R, Nickel B, Hersch J, et al. What do you think overdiagnosis means? A qualitative analysis of responses from a national community survey of Australians. BMJ Open 2015;5:e007436.

27 Moynihan R, Sims R, Hersch J, et al. Communicating about overdiagnosis: learning from community focus groups on osteoporosis. PLoS One 2017;12:e0170142.

28 Hersch J, McGeechan K, Barratt A, et al. How information about overdetection changes breast cancer screening decisions: a mediation analysis within a randomised controlled trial. BMJ Open 2017;7:e016246.

29 Shepherd HL, Barratt A, Trevena LJ, et al. Three questions that patients can ask to improve the quality of information physicians give about treatment options: a cross-over trial. Patient Educ Couns 2011;84:379-85.

30 Slade SC, Kent P, Patel S, et al. Barriers to primary care clinician adherence to clinical guidelines for the management of low back pain: a systematic review and Metasynthesis of qualitative studies. Clin J Pain 2016;32:800-16.

31 Pines JM, Isserman JA, Szyld D, et al. The effect of physician risk tolerance and the presence of an observation unit on decision making for ED patients with chest pain. Am J Emerg Med 2010;28:771-9.

32 Elwyn G, Frosch D, Thomson R, et al. Shared decision making: a model for clinical practice. J Gen Intern Med 2012;27:1361-7.

33 Born KB, Levinson W. Choosing wisely campaigns globally: a shared approach to tackling the problem of overuse in healthcare. $J$ Gen Fam Med 2019;20:9-12.

34 Moynihan R, Nickel B, Hersch J, et al. Public opinions about overdiagnosis: a national community survey. PLoS One 2015;10:e0125165.

35 Nickel B, Semsarian C, Moynihan R, et al. Public perceptions of changing the terminology for low-risk thyroid cancer: a qualitative focus group study. BMJ Open 2019;9:e025820.

36 Cardinal JS, Gunderman RB, Tarver RD. Informing patients about risks and benefits of radiology examinations: a review article. J Am Coll Radiol 2011;8:402-8.

37 Woloshin S, Schwartz LM. Communicating data about the benefits and harms of treatment: a randomized trial. Ann Intern Med 2011;155:87-96.

38 Jessup RL, Buchbinder R. What if I cannot choose wisely? Addressing suboptimal health literacy in our patients to reduce overdiagnosis and overtreatment. Intern Med J 2018;48:1154-7.

39 Toledo-Chávarri A, Rué M, Codern-Bové N, et al. A qualitative study on a decision aid for breast cancer screening: views from women and health professionals. Eur J Cancer Care 2017;26:e12660.

40 Stacey Det al. Decision AIDS for people facing health treatment or screening decisions. Cochrane Database Syst Rev 2014;1.

41 Ghanouni A, Renzi C, Waller J. Improving public understanding of 'overdiagnosis' in England: a population survey assessing familiarity with possible terms for labelling the concept and perceptions of appropriate terminology. BMJ Open 2018;8:e021260.

42 Chen JY, Eborall H, Armstrong N. Stakeholders' positions in the breast screening debate, and media coverage of the debate: a qualitative study. Crit Public Health 2014;24:62-72.

43 Nagler RH, Fowler EF, Marino NM, et al. The evolution of mammography controversy in the news media: a content analysis of four Publicized screening recommendations, 2009 to 2016. Womens Health Issues 2019;29:87-95.

44 O'Keeffe M, Maher CG, Stanton TR, et al. Mass media campaigns are needed to counter misconceptions about back pain and promote higher value care. Br J Sports Med 2019;53:1261-2.

45 Zikmund-Fisher BJ, Kullgren JT, Fagerlin A, et al. Perceived Barriers to Implementing Individual Choosing Wisely ${ }^{\circledR}$ Recommendations in Two National Surveys of Primary Care Providers. J Gen Intern Med 2017;32:210-7.

46 Bhatia RS, Levinson W, Shortt S, et al. Measuring the effect of choosing wisely: an integrated framework to assess campaign impact on low-value care. BMJ Qual Saf 
47 Jenniskens K, de Groot JAH, Reitsma JB, et al. Overdiagnosis across medical disciplines: a scoping review. BMJ Open 2017;7:e018448.

48 Born KB, Coulter A, Han A, et al. Engaging patients and the public in choosing wisely. BMJ Qual Saf

49 Shamseer L, Moher D, Clarke M, et al. Preferred reporting items for systematic review and meta-analysis protocols (PRISMA-P) 2015: elaboration and explanation. BMJ 2015;349:g7647.

50 Harris JL, Booth A, Cargo M, et al. Cochrane qualitative and implementation methods group guidance series-paper 2: methods for question formulation, searching, and protocol development for qualitative evidence synthesis. J Clin Epidemiol 2018;97:39-48.

51 Higgins JPT, Green S. Cochrane Handbook for Systematic Reviews of Interventions. Cochrane, 2011.

52 Frandsen TF, Gildberg FA, Tingleff EB. Searching for qualitative health research required several databases and alternative search strategies: a study of coverage in bibliographic databases. J Clin Epidemiol 2019;114:118-24.

53 Wong SS-L, Wilczynski NL, Haynes RB, et al. Developing optimal search strategies for detecting clinically relevant qualitative studies in MEDLINE. Stud Health Technol Inform 2004;107:311-6.

54 McKibbon KA, Wilczynski NL, Haynes RB. Developing optimal search strategies for retrieving qualitative studies in PsycINFO. Eval Health Prof 2006;29:440-54.

55 Moher D, Liberati A, Tetzlaff J, et al. Preferred reporting items for systematic reviews and meta-analyses: the PRISMA statement. PLoS Med 2009;6:e1000097.

56 The Critical Appraisals Skills Programme. Casp qualitative checklist 2018, 2018.

57 Thomas J, Harden A. Methods for the thematic synthesis of qualitative research in systematic reviews. BMC Med Res Methodol 2008;8:45.

58 McAllum K, Fox S, Simpson M, et al. A comparative tale of two methods: how thematic and narrative analyses author the data story differently. Communication Research and Practice 2019;5:358-75.

59 Booth A, Noyes J, Flemming K, et al. Guidance on choosing qualitative evidence synthesis methods for use in health technology assessments of complex interventions, 2016.

60 Barnett-Page E, Thomas J. Methods for the synthesis of qualitative research: a critical review. BMC Med Res Methodol 2009;9:59.

61 Miles MBet al. Qualitative data analysis: An expanded sourcebook. sage, 1994
62 Bengtsson M. How to plan and perform a qualitative study using content analysis. NursingPlus Open 2016;2:8-14.

63 Dixon-Woods M, Agarwal S, Jones D, et al. Synthesising qualitative and quantitative evidence: a review of possible methods. $J$ Health Serv Res Policy 2005;10:45-53.

64 Rozbroj T, Lyons A, Lucke J. The Mad leading the blind: perceptions of the vaccine-refusal movement among Australians who support vaccination. Vaccine 2019;37:5986-93.

65 Clancy M. Is reflexivity the key to minimising problems of interpretation in phenomenological research? Nurse Res 2013;20:12-16.

66 Lewin S, Bohren M, Rashidian A, et al. Applying GRADE-CERQual to qualitative evidence synthesis findings - paper 2: how to make an overall CERQual assessment of confidence and create a summary of qualitative findings table. Implementation Science 2018;13.

67 Noyes J, Booth A, Lewin S, et al. Applying GRADE-CERQual to qualitative evidence synthesis findings-paper 6: how to assess relevance of the data. Implementation Science 2018;13.

68 Glenton C, Carlsen B, Lewin S, et al. Applying GRADE-CERQual to qualitative evidence synthesis findings - paper 5 : how to assess adequacy of data. Implementation Science 2018;13.

69 Colvin CJ, Garside R, Wainwright M, et al. Applying GRADECERQual to qualitative evidence synthesis findings-paper 4: how to assess coherence. Implementation Science 2018;13.

70 Tong A, Flemming K, Mclnnes $\mathrm{E}$, et al. Enhancing transparency in reporting the synthesis of qualitative research: ENTREQ. BMC Med Res Methodol 2012;12:181.

71 Gordon EC. Understanding in Epistemology. N.D, 2019. Available: https://www.iep.utm.edu/understa/\#H7 [Accessed 22 Oct 2019].

72 O'Grady LA, Witteman $\mathrm{H}$, Wathen CN. The experiential health information processing model: supporting collaborative web-based patient education. BMC Med Inform Decis Mak 2008;8:58.

73 Tyng CM, Amin HU, Saad MNM, et al. The influences of emotion on learning and memory. Front Psychol 2017;8:1454.

74 McCoy MS, Warsh J, Rand L, et al. Patient and public involvement: two sides of the same coin or different coins altogether? Bioethics 2019;33:708-15.

75 Berlin L. Overdiagnosed: making people sick in pursuit of health. JAMA 2011;305:1356-9.

76 Temkin SM. "Less is more:" The harms of overtreatment in early ovarian cancer. Gynecol Oncol 2015;137:191-2. 